\title{
IMPORTANCIA DE LA EVALUACIÓN DEL NEURODESARROLLO EN PACIENTES PEDIÁTRICOS CON HEMOFILIA
}

\author{
Blanca Estela Huitrón Vázquez, Carlos Prado Romero,Maricela Osorio Guzmán \\ y Georgina Eugenia Bazán Riverón \\ UNAM \\ México
}

\begin{abstract}
RESUMEN
La psicología del desarrollo se define como el estudio científico de los cambios que ocurren en las personas a lo largo de la vida; dichos cambios pueden verse afectados por las enfermedades crónicas, las cuales inciden en aspectos biológicos, psicológicos y sociales de quienes las padecen, como es el caso de la "hemofilia". El objetivo de esta investigación es presentar los resultados de la evaluación de cinco pacientes pediátricos con hemofilia, para identificar sus habilidades de desarrollo, las áreas de oportunidad o zona real de desarrollo, y plantear/sugerir actividades a los cuidadores primarios para elevar sus competencias y/o mantener la calidad de vida de los niños; los instrumentos aplicados fueron la prueba de escrutinio del desarrollo de Denver II (Frankerburg y Dodds, 1990) y la prueba valoración de la conducta refleja y reacciones de defensa (Huitrón y Alvarado, 1998). Los resultados revelan que los participantes mostraron resultados diferentes en su desarrollo aun cuando mostraron tener los reflejos y reacciones de defensa esperados para su edad. Se concluye que en los participantes el padecer una enfermedad crónica que impacta su desarrollo cobra importancia fundamental la atención que se debe dar de manera interprofesional, involucrando a la familia y a la comunidad.
\end{abstract}

Palabras Clave:

Denver II, desarrollo infantil, educación temprana personalizada, hemofilia

\section{IMPORTANCE OF NEURODEVELOPMENTAL EVALUATION IN PEDIATRIC PATIENTS WITH HEMOPHILIA}

\begin{abstract}
The psychology of development is definite as the scientific study of the changes that occur in people throughout life, chronic diseases, which affect biological, psychological and social aspects of those who suffer, can affect these changes, such is the case of "hemophilia". The objective of this research is present the results of the evaluation of 5 pediatric patients with hemophilia, to identify their development skills, opportunity areas or real development area and propose/ suggest activities to primary caregivers to raise their competences and to maintain the children quality of life. The instruments applied were "The test of scrutiny of development by Denver II" (Frankerburg \& Dodds, 1990) and the test "assessment of the reflect behavior and defense reactions (Huitrón \& Alvarado, 1998). The results expose that the participants showed different results in their development even when they showed to have the reflexes and defense reactions expected for their age. It concludes that, in the participants suffering a chronic disease that affects their development, takes on fundamental importance, the attention that must give in an interprofessional way, involving the family and the community.
\end{abstract}

Keywords:

Denver II, child development, personalized early education, hemophilia

Bitácora del Artículo:

| Recibido: 1 de Febrero de 2019 | Aceptado: 1 Abril de 2019 | Publicado en línea: Enero-Junio de 2019 | 


\title{
IMPORTANCIA DE LA EVALUACIÓN DEL NEURODESARROLLO EN PACIENTES PEDIÁTRICOS CON HEMOFILIA
}

\author{
Blanca Estela Huitrón Vázquez, Carlos Prado Romero,Maricela Osorio Guzmán \\ y Georgina Eugenia Bazán Riverón \\ UNAM \\ México
}

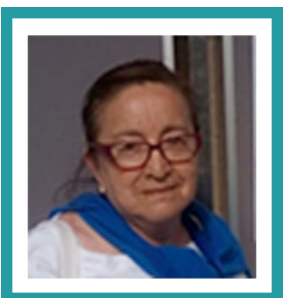

Blanca Estela Huitrón Vázquez

FES-Iztacala, UNAM

Correo: huvbmx@yahoo.es

Doctora en Psicología evolutiva y de la Educación por la Universidad de Sevilla. Es miembro titular de Asociaciones Profesionales de Psicología. Profesor titular "B" de tiempo completo de la carrera de Psicología en FES-Iztacala, UNAM.

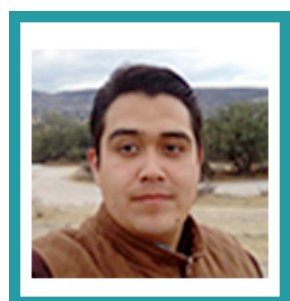

Carlos Prado Romero

FES-Iztacala, UNAM

Correo: carlosprador9318@gmail.com

Estudiante de Doctorado en Psicología y Salud en la UNAM. Colaborador activo en el grupo de investigación "Enfermedades crónicas"; y voluntario en la Federación de Hemofilia de la República Mexicana.

\section{Contribución de los Autores}

Blanca Estela Huitón estuvo a cargo de la elaboración del marco teórico, aplicación de los instrumentos, análisis y redacción de datos, conclusiones y referencias. I Carlos Prado participó en la elaboración del marco teórico, redacción de datos, conclusiones y referencias. | Maricela Osotio realizó la concepción, elaboración del marco teórico, método, análisis y redacción de datos, conclusiones y referencias. I Georgina Bazán estuvo a cargo del análisis y redacción de datos, revisión de conclusiones y referencias.

\section{AGRADECIMIENTOS}

Se agradece a todas aquellas personas que participaron e hicieron posible la realización de este estudio.

\section{DATOS DE FILIACIÓN DE LOS AUtORES}

Facultad de Estudios Superiores Iztacala

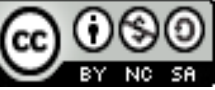

Copyright: (c) 2019 Huitrón-Vázquez, B. E.; Prado-Romero, C.; Osorio-Guzmán, M.; \& Bazán-Riverón, G.E.

Este es un artículo de acceso abierto distribuido bajo los términos de la licencia Creative Commons Reconocimiento-NoComercial 4.0 Internacional, por lo que su contenido gráfico y escrito se puede compartir, copiar y redistribuir total o parcialmente sin necesidad de permiso expreso de sus autoras con la única condición de que no se puede usar con fines directamente comerciales y los términos legales de cualquier trabajo derivado deben ser los mismos que se expresan en la presente declaración. La única condición es que se cite la fuente con referencia a la Revista Digital Internacional de Psicología y Ciencia Social y a sus autoras. 


\section{TABLA DE CONTENIDO}

INTRODUCCIÓN

MÉTOdO

Participantes , 25

Procedimiento, 25

Mediciones , 25

Análisis estadísticos, 26

RESULTADOS

Participante 1 (44 meses) , 26

Participante 2 ( 7 meses) , 26

Participante 3 (23 meses) , 26

Participante 4 (42 meses) , 27

Participante 5 (60 meses) , 27

Recomendaciones , 28

Discusión

CONCLUSIONES 


\section{INTRODUCCIÓN}

\section{T}

a psicología del desarrollo se define como "el estudio científico de los cambios que ocurren en las personas a lo largo de la vida" (Berger, 2001), es decir, el estudio sistematizado de los cambios psicológicos, fisiológicos y conductuales (Novak, 1998) determinados por factores genéticos, ambientales y socioculturales (Tirado, Martínez, Cobarrubias, López, Quezada, Olmos y Díaz-Barriga, 2010).

A través del tiempo se han desarrollado diferentes enfoques, los cuales intentan explicar si el desarrollo se debe prevalentemente a la herencia genética, al ambiente o a una mezcla de ellos, además de brindar parámetros acerca de la normalidad de la conducta a diversas etapas del desarrollo. Entre éstos se pueden identificar el enfoque genético (Gesell, 1985), el conductual (Watson, 1925, como se citó en Ardila, 2013; León, 1984; Papalia, Wendkos y Duskin, 2009), el constructivista (Piaget, 1991, como se citó en Montealegre, 2016; Houdé, 2006) y el culturalista (Vigotsky, 1987, como se citó en Montealegre, 2016; Rogoff, 1993), entre los más importantes.

Estos enfoques enlistan una serie de factores previos al embarazo, durante el mismo, en el momento del parto y posterior a éste, además de los relacionados con el contexto o sistemas en que se desarrolla el niño que, en conjunto, determinan un desarrollo óptimo o uno deficiente (Bronfenbrenner, 1987, como se citó en Gifre y Esteban, 2012). Entre los factores de riesgo están, por ejemplo, la edad de la madre: si es muy joven puede presentar problemas debido a la "inmadurez pelviana" (Roberts, 1982; Manual CTO de Medicina y Cirugía, 2016) ; si las mujeres son mayores de 35 años tienen mayor posibilidad de desarrollar diabetes gestacional - la alteración metabólica más frecuente-; el consumo de sustancias toxicas (como el tabaco, alcohol y/o drogas) (Reece, 2010). A su vez, durante el embarazo, aumenta la probabilidad de padecer infecciones en vías urinarias, que pueden contribuir a una interrupción del embarazo o a un parto prematuro; otro factor es el aumento excesivo de peso de la madre que, aunado con la edad de sus óvulos, incrementa el riesgo de aneludopatías (como el síndrome de Turner o de Down) (Craig, 2001).

Otro aspecto importante es la alimentación de la madre, porque al presentar desnutrición durante el embarazo se incrementa la probabilidad de una disminución en el desarrollo del cerebro del producto, lo cual podría alterar las capacidades intelectuales (Reece, 2010) o, en caso contrario, al padecer obesidad también, se incrementa el riesgo de macrosomía fetal, diabetes gestacional o bajo peso para la edad gestacional, malformaciones genéticas y aumento de riesgo de muerte fetal (Lozano, Betancourt, Turcios, Cueva, Ocampo, Portillo y Lozano, 2016). Aunado a lo anterior, Acosta, Ramos, Zamora y Murillo (2014) mencionan algunos factores ambientales (como la escolaridad, bajo nivel de ingresos familiares, zonas sin servicios o zonas contaminadas) como factores que potencian el riesgo en el desarrollo de los infantes.

Por lo expuesto, es fundamental formar equipos de salud interprofesional capacitados en la detección de estos factores, efectuando una evaluación e intervención temprana personalizada, que informe el desarrollo del individuo, detectando los retrasos o alteraciones que se puedan presentar a lo largo de su proceso de maduración. Así, Gómez, Viguer y Cantero (2013) y Chávez (2003) refieren que la atención temprana tiene como finalidad ofrecer a los niños con déficit o con riesgo de padecerlos, un conjunto de acciones optimizadoras y compensadoras que faciliten su adecuada maduración en todos los ámbitos y que les permita optimizar el desarrollo durante los primeros seis años de vida; ya que los individuos que reciben mayor apoyo y acompañamiento por parte de su entorno familiar presentarán mayor desarrollo personal y de integración social (Torralva, Cugnasco, Manso, Sauton, Ferrero, O'donnell, Durán y Carmuega, 1999).

Por otra parte, desafortunadamente muchos niños nacen con algún padecimiento ; las enfermedades crónicas infantiles son un grupo heterogéneo de padecimientos con distintas características. En su mayoría, las enfermedades son progresivas y causan deterioro físico importante, perjudican el desarrollo del niño y requieren tratamientos dolorosos, invasivos y/o largas hospitalizaciones (Eiser, 1996; Garralda, 1994; Perrin y Shonkoff, 2000).

Aunque los niños sanos y los enfermos tienen que superar las mismas tareas evolutivas, para los últimos es más complicado debido a la influencia de los aspectos fisiológicos de la propia enfermedad, tratamiento, hospitalizaciones, limitaciones físicas y alteraciones en las relaciones familiares (Midence, 1994; Blum, Gordillo y García, 2003; Pedreira, 1998; Osorio, Olvera, Bazán y Gaitán, 2016).

Una de las enfermedades crónicas de interés para la psicología es la hemofilia, entre otras razones por las graves consecuencias del padecimiento y porque se alteran los aspectos biológicos, psicológicos y sociales (Osorio, 2014). La hemofilia es una enfermedad hemorrágica ligada al cromosoma " $X$ ", caracterizada por la deficiencia funcional o cuantitativa de los factores de coagulación VIII para la hemofilia "A" o IX para la hemofilia "B". La 
incidencia de la misma en la población mundial se estima en 1 de cada 10,000 varones nacidos vivos. La hemofilia se distribuye uniformemente en la población y la Federación Mundial de Hemofilia (fmh, 2012) estima que a nivel mundial hay 400,000 personas con esta coagulopatía.

El momento del diagnóstico en un niño con una enfermedad crónica como la hemofilia, es vivido por la mayoría de las familias como una situación traumática, registrándose la aparición de altos niveles de estrés y alteraciones psicológicas y emocionales. En parte esto se debe al cambio de la rutina general y a la pérdida de control de la situación familiar por parte de los padres, así como a la incertidumbre derivada de la enfermedad misma (Arranz, Costa, Bayés, Cancio, Magallón y Fernández, 2000; Goldstein, Golan, Kenet y Martinowitz, 2000; Graña y Rodríguez, 2000; Osorio, 2016). En cuanto al desarrollo de los niños, las actividades normales se ven restringidas por el miedo a las hemorragias, y los cuidadores no permiten la autonomía de los menores, restringen la socialización, no les permiten gatear, caminar o saltar, como lo haría cualquier otro infante, lo que en muchas ocasiones los lleva a presentar retrasos en su desarrollo normal. En estos casos es frecuente encontrar niños sobreprotegidos.

Por lo anterior, el objetivo de esta investigación es presentar los resultados de la evaluación de cinco pacientes pediátricos con hemofilia, para identificar sus habilidades de desarrollo, las áreas de oportunidad o zona real de desarrollo, y plantear/sugerir actividades a los cuidadores primarios para elevar sus competencias y/o mantener la calidad de vida de los niños, debido a que al momento de desarrollar esta investigación no se encontraron en la literatura mundial trabajos referentes a esta temática.

\section{Método}

\section{Participantes}

Cinco pacientes pediátricos varones con hemofilia, con una media de edad de 35.2 meses, DT $=20.51$, seleccionados a partir de muestreo no probabilistico por conveniencia (Shaughnessy, Zechmeister y Zechmeister, 2007) ; 60\% de ellos tiene diagnóstico de hemofilia tipo A y el $40 \%$ restante hemofilia B; el grado clínico de todo el grupo es severo.

Todos los padres firmaron el consentimiento informado, asistieron a la sesión de evaluación, recibieron los informes y sugerencias de atención.

\section{Procedimiento}

En el día mundial de la Hemofilia se invitó a la comunidad de pacientes y padres con hemofilia, por medio de la Federación de Hemofilia de la República Mexicana, a la Jornada Integral de Salud, en la Facultad de Estudios Superiores Iztacala, unam; los niños, acompañados de sus madres y/o padres, asistieron al módulo del cietep, donde un grupo interprofesional los evaluó; se revisaron las pruebas aplicadas, se eliminaron las evaluaciones incompletas, y se analizaron y estructuraron los informes. El criterio de inclusión fue la edad de los pacientes, los cuales deberían ser menores de 6 años.

\section{Mediciones}

Se aplicó la Prueba de Escrutinio del Desarrollo de Denver II (Frankerburg y Dodds, 1990); el objetivo de esta escala es la detección e intervención temprana de deficiencias en el desarrollo, confirmar o descartar diferentes sospechas de alteraciones y dar seguimiento a niños de alto riesgo. Las edades en que se aplica son desde el primer mes hasta los 60 meses de edad y evalúa las siguientes áreas.

1. Personal social. Se refiere a la socialización y autonomía del niño.

2. Motor fino adaptativo. Capacidad del niño de coordinar ojo-mano y solucionar problemas.

3. Lenguaje. Habilidad del niño para comprender y expresarse de manera verbal.

4. Motricidad gruesa. Capacidad de los músculos grandes, como arrastrarse, gatear, sentarse, caminar o deambular y saltar, entre otros.

Las categorías diagnósticas que ofrece son las siguientes.

1. Desarrollo normal. No hay retraso; puede efectuar correctamente todas las conductas de su edad.

2. Desarrollo dudoso. Se presenta al menos un retraso en una conducta y dos o más actividades de cuidado (fallas o retrasos en actividades a desarrollar en su edad).

3. Desarrollo anormal. Dos a más retrasos.

4. Inaceptable (rehúso). Actividades que el niño rehusó efectuar y que, de haberlas fallado, habrían sido interpretadas como retraso.

Además de la prueba valoración de la conducta refleja y reacciones de defensa (Huitrón y Alvarado, 1998). El objetivo de esta prueba es evidenciar el estado de maduración neurológica del niño. Aplicable desde el primer mes hasta los 24 meses, evaluando los reflejos con que se nace, cómo se van integrando de acuerdo con la edad y las reacciones de defensa que permanecen, para el resto de su vida, en el repertorio de los participantes. 


\section{Análisis estadísticos}

Se efectuó un análisis de tipo descriptivo, presentando los casos de cada uno de los participantes.

\section{Resultados}

Los datos se presentan de acuerdo con cada una de las áreas que conforman los instrumentos, iniciando por las habilidades que tiene el niño y las áreas de oportunidad, mencionando después las sugerencias de actividades a trabajar junto con el cuidador principal.

\section{Participante 1 (44 meses)}

Al inicio de la evaluación, el pequeño se mostró intranquilo; contenido por la mamá, respondió con interés ante los materiales y actividades de la prueba, lo que permitió desarrollar la evaluación, obteniendo los siguientes resultados.

Personal social. Respecto a las conductas de autocuidado fue capaz de ponerse algunas prendas (calcetines y calzones); aún se le dificulta ponerse la camiseta, lavarse y secarse las manos; puede cepillarse los dientes y prepararse cereal con supervisión; puede jugar cartas, seguir reglas y esperar turnos; fue incapaz de mencionar el nombre de algún amigo.

Motricidad fina. Puede hacer una torre de ocho cubos, mover su dedo pulgar independientemente del resto de los dedos, dibujar tres partes de una persona, elegir de modo visual la línea más larga entre dos. Se le dificulta imitar una línea vertical.

Lenguaje. Es capaz de identificar y nombrar cuatro dibujos de cinco (gato, caballo, paloma, perro y hombre), expresar el uso de tres objetos cotidianos, expresar qué hacer si tiene alguna necesidad (frío, hambre), nombrar cuatro colores, adjetivos y define cinco palabras (como pelota, casa y techo, entre otras).

Motricidad gruesa. Puede aventar una pelota por arriba de su cabeza, saltar de un lado a otro, incluso con longitud, así como conservar el equilibrio en cada pie durante cinco segundos.

De acuerdo con los resultados de las pruebas aplicadas, se concluye que en la Escala de Escrutinio del Desarrollo de Denver II su desarrollo es "Anormal" y no corresponde a las competencias de su edad, presentando cuatro retrasos, sobre todo en las áreas motora fina y personal social.

Respecto a la conducta refleja y reacciones de desarrollo, las mismas se encuentran integradas.

\section{Participante 2 (7 meses)}

Durante la evaluación el pequeño se mostró tranquilo, sonriente y cooperador; efectuó las actividades sin problema, con buena disposición. Presentó las siguientes habilidades en cada una de las siguientes áreas.

Personal social. Mira con atención un objeto y lo alcanza, come por sí mismo llevando pequeños alimentos a su boca.

Motricidad fina. Sigue objetos con la vista, toma una pasa en movimiento de rastrillo, logra alcanzar y pasar un objeto de una mano a otra y come por sí mismo.

Lenguaje. Al escuchar su nombre vuelve hacia el origen de la voz o de un sonido; expresa sílabas sencillas: na, ta, ma, pa.

Motricidad gruesa. Voltea hacia ambos lados (de posición prona a supina, y viceversa); al Ilevarlo sentado, la cabeza sigue al cuerpo; puede sentarse con buen equilibrio sin apoyo.

De acuerdo con los resultados de las pruebas aplicadas, se concluye que en la Escala de Escrutinio del Desarrollo de Denver II su desarrollo es "Normal", obteniendo un solo cuidado - conducta que no puede efectuar, pero que aún tiene tiempo para adquirir-, el cual es agarrar dos cubos.

Respecto a la conducta refleja, ésta se está integrando de modo adecuado para dar paso a nuevos patrones de desarrollo, y en cuanto a las reacciones de defensa, éstas se encuentran en proceso de consolidación.

\section{Participante 3 (23 meses)}

Se mostró tranquilo y cooperador durante la evaluación; desarrolló las actividades sin problema, obteniendo los siguientes resultados.

Personal social. Es capaz de ayudar en pequeñas tareas de casa, usar cuchara y tenedor para alimentarse, dar de comer a un muñeco, y lavarse y secar las manos por completo. Se le dificulta quitarse y ponerse prendas sencillas de ropa y cepillarse los dientes por sí mismo, por lo que es importante ayudarle para que adquiera conductas de autocuidado, iniciando por quitarse las prendas de vestir para después vestirse con supervisión y luego sin ella.

Motricidad fina. Puede hacer una torre de cuatro cubos y sacar objetos o alimentos pequeños de un frasco con boca estrecha.

Lenguaje. Es capaz de indicar de dos a cuatro dibujos cotidianos, hablar combinando dos palabras con un lenguaje entendible, e identificar y nombrar seis partes de su cuerpo 
Motricidad gruesa.Puede correr y aventar una pelota hacia adelante; se le dificulta subir una escalera por sí mismo y saltar con ambos pies en un mismo lugar.

De acuerdo con los resultados de las pruebas aplicadas, se concluye que en la Escala de Escrutinio del Desarrollo de Denver II su desarrollo es "Dudoso", pues se le dificulta quitarse prendas de ropa y subir escaleras por sí mismo.

\section{Participante 4 (42 meses)}

El niño se mostró sonriente, tranquilo y cooperador durante la evaluación; efectuó todas las actividades y contestó todas las preguntas de la evaluadora, obteniendo los siguientes resultados.

Personal social. Fue capaz de vestirse, cepillarse los dientes y prepararse cereal sin ayuda; se le dificulta desarrollar un juego con reglas y turnos.

Motricidad fina. Pudo hacer una torre de ocho cubos, imitar movimientos digitales, dibujó una persona con tres partes, eligió adecuadamente una línea más larga, pudo imitar una línea vertical, y hacer un círculo y una cruz.

Lenguaje. Fue capaz de expresar el uso de tres objetos cotidianos, expresar qué hacer si tiene alguna necesidad (frío, hambre), expresar qué sonidos hacen cuatro animales, nombrar cuatro colores y contar un cubo. Se le dificulta entender cuatro preposiciones de lugar.

Motricidad gruesa. Puede aventar una pelota por arriba de su cabeza, saltar de un lado a otro y guardar el equilibrio en cada pie sólo durante dos segundos. Se le dificulta saltar en un solo pie.

De acuerdo con los resultados de la prueba, se concluye que en la Escala de Escrutinio del Desarrollo de Denver II el desarrollo de este niño es "Normal" y que corresponde a su edad.

Respecto a la conducta refleja y reacciones de desarrollo, éstas se encuentran integradas.

\section{Participante 5 (60 meses)}

Durante la evaluación, el pequeño se mostró inquieto, mantuvo un periodo de atención corto al desempeñar las actividades, y en ocasiones fue necesario repetir las instrucciones de la tarea para que las efectuara, obteniendo los siguientes resultados.

Personal social. Fue capaz de vestirse sin ayuda, jugar un juego con reglas y turnos, y cepillarse los dientes por sí mismo. Se le dificultó preparar un alimento sencillo.

Motricidad fina. Pudo copiar un círculo y una cruz, dibujar una persona de tres partes e identificar una línea más larga entre dos. Fue capaz de copiar un círculo y una cruz, un cuadrado con demostración y dibujar una persona de tres partes, actividad importante para la adquisición del esquema corporal, escoger una línea más larga entre dos; se le dificultó copiar un cuadrado con demostración y dibujar una persona de seis partes.

Lenguaje. Es capaz de expresarse de manera entendible, logra identificar qué sonidos emiten cuatro animales, entender cuatro preposiciones de lugar, nombrar cuatro colores y expresar qué hacer si tiene alguna necesidad (frío, hambre o sueño). Se le dificulta definir cinco objetos, contar cubos y nombrar dos adjetivos opuestos.

Motricidad gruesa. Puede saltar en un pie y guardar el equilibrio en cada pie durante tres segundos. Se le dificulta equilibrarse en cada pie durante cuatro o más segundos y caminar de punta-talón, colocando el talón delante del pie y tocando el dedo del otro pie.

De acuerdo con los resultados de las pruebas aplicadas, se concluye que en la Escala de Escrutinio del Desarrollo de Denver II su desarrollo es "Dudoso" porque se le dificulta preparar un alimento sencillo, copiar un cuadrado cuando se le muestra cómo hacerlo, expresar adjetivos opuestos y guardar el equilibrio durante cuatro segundos o más.

Respecto a la conducta refleja y reacciones de desarrollo, se encuentran integradas.

Para resumir lo expuesto, en la tabla 1 se muestran los resultados. Como se observa, en la prueba Denver II sólo un participante fue "Anormal", lo cual indica dos a más retrasos en conductas que ya deberían estar en su repertorio. Respecto a los clasificados como dudosos, presentaron al menos un retraso en una conducta y dos o más actividades de cuidado; por último, dos niños obtuvieron resultados dentro de su rango de edad.

\section{Tabla 1.}

Resultados de las pruebas aplicadas a los participantes.

\begin{tabular}{lcclll} 
Paciente & $\begin{array}{c}\text { Edad } \\
\text { (Meses) }\end{array}$ & $\begin{array}{c}\text { Tipo de } \\
\text { Hemofilia }\end{array}$ & Denver II & Reflejos \\
\hline Sujeto 1 & 44 & A & Anormal & $24 \mathrm{~m}$ \\
Sujeto 2 & 7 & A & Normal & $8 \mathrm{~m}$ \\
Sujeto 3 & 23 & B & Dudoso & $24 \mathrm{~m}$ \\
Sujeto 4 & 42 & A & Normal & $24 \mathrm{~m}$ \\
Sujeto 5 & 60 & B & Dudoso & $24 \mathrm{~m}$
\end{tabular}

Para observar los resultados por área se elaboró la figura 1, donde se puede identificar con claridad el desempeño de cada uno de los participantes. 


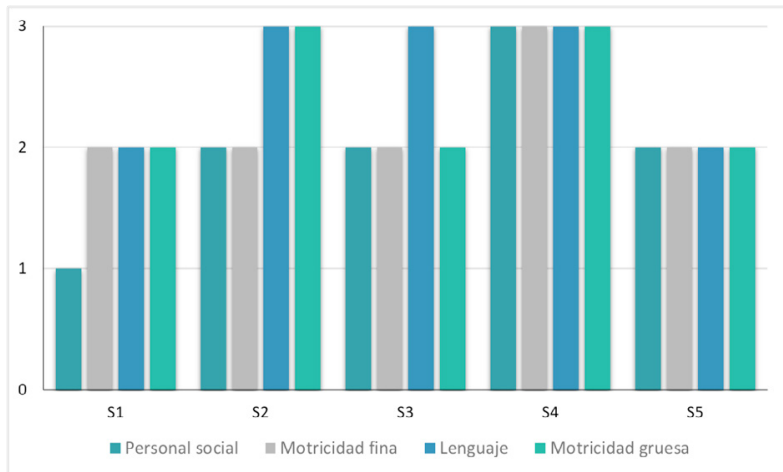

1=anormal, 2=dudoso, 3=normal

Figura 1.

Resultados de cada participante por área de la prueba Denver II.

\section{Recomendaciones}

Siguiendo los objetivos de la educación temprana que estipula que las evaluaciones son personalizadas, que el desarrollo es céfalocaudal, próximo distal y de lo general a lo específico, y que el desarrollo inicia desde la concepción hasta la muerte, se hicieron diferentes recomendaciones, dependiendo de las habilidades con que contaba en ese momento el niño; entre las más comunes destacaron las siguientes.

Personal social. Promover esta área es función primordial de los cuidadores principales, iniciando con mirar a la cara, responder con sonrisas, además de enseñar conductas como saludar o conductas de autocuidado (como el lavado de dientes).

Motricidad fina. El contacto y el seguimiento visual son los inicios de esta área; agarrar el sonajero requiere que haya integrado el reflejo palmar, para después hacerlo de manera voluntaria; , fortalecer el movimiento de pinza fina, mediante diferentes actividades, pasar objetos pequeños de una mano a otra, chocar ambos objetos, hacer bolitas de papel china con tiras de papel bond. Realizar diferentes líneas o dibujos que impliquen hacer líneas rectas, perpendiculares, círculos o cuadrados, iniciando por garabateo, competencias previas para la lectura y la escritura.

Lenguaje. Estas conductas inician con vocalizar o hacer ruidos guturales, por lo que es importante apoyar el desarrollo del lenguaje mediante diálogos entre el cuidador y el pequeño, o entre iguales, así como con diferentes juegos verbales donde se pronuncian o repiten silabas apoyados de movimientos rítmicos (como palmadas). Preguntar de modo directo a los niños acerca del uso de diferentes objetos apoyados (visualmente) de los mismos.

Motricidad gruesa. Estas habilidades inician desde la integración de los reflejos primarios, presentando movimientos simétricos, la capacidad para controlar la cabeza, el arrastre, el gateo y por supuesto capaz de sentarse por sí solo y caminar; para todo ello, el juego es fundamental. Se recomienda el trabajo con pelotas (aventar y patear), realizar juegos de posturas diversas o brincar sobre un pie y promover el equilibrio.

\section{Discusión}

Efectuar investigaciones como la presente permitirá poder intervenir con pacientes con algún padecimiento crónico y detectar a tiempo retrasos en su desarrollo y variables de riesgo que alteren la adquisición de conductas normativas.

Es importante mencionar que se considera la evaluación como un proceso, y que no se deben olvidar de manera ecológica todos los factores y contextos involucrados. De esta manera, el estudio del desarrollo de los niños —según Bronfenbrenner (1987)— debe de incluir el análisis de los diferentes niveles contextuales que los rodean, esto es:

- Microsistema. Complejo de interacciones del entorno inmediato.

- Mesosistema. Interconexión de entornos; un conjunto de relaciones entre dos o más entornos, en los que la persona participa de manera activa.

- Exosistema. Comprende uno o más entornos que no incluyen a la persona en desarrollo como participante activo, pero en los que se producen hechos que le afectan o se ven afectados.

- Macrosistema. Patrones ideológicos e instituciones sociales comunes de la cultura en que el niño se encuentra inmerso.

Según esta postura, el desarrollo es el proceso por el cual la persona adquiere una concepción del ambiente ecológico más amplia, diferenciada y válida, se motiva y se torna capaz de desarrollar actividades que revelen propiedades de ese ambiente, en diferentes niveles de complejidad. Aquí se asume a la persona como un ser activo, "una entidad creciente, dinámica, que va adentrándose progresivamente y reestructurando el medio en que vive" (Bronfenbrenner, 1987). En el caso aquí analizado, con pacientes que pueden tener alteraciones propias del padecimiento (como hemorragias constantes y artropatías, entre otras), analizar todos los niveles contextuales es fundamental para dar recomendaciones especializadas, personalizadas y dirigidas a campos de oportunidad específicos para lograr un desarrollo óptimo, normal; el uso de pruebas de tamiz son eficientes para una detección oportuna y poder sugerir a los cuidadores principales actividades acordes a las áreas de 
oportunidad requeridas por cada uno de los niños evaluados; sin embargo, no hay que olvidar que también es fundamental utilizar pruebas que complementen la información, como las pruebas que proporcionan un coeficiente de desarrollo (Bayley, 1977, 1993) o las que confieran información psicomotriz (McCarthy, 1991). Por último, considerar la evaluación como un proceso es fundamental para hacer una valoración posterior a la implementación de las sugerencias proporcionadas.

\section{CONCLUSiOnes}

De esta manera cobra importancia fundamental la atención que de manera interprofesional se debe proporcionar al niño que padece hemofilia, sin olvidar a la familia y a la comunidad.

Es necesario que haya detección oportuna, apoyo y seguimiento al grupo familiar en todas las áreas de desarrollo, porque es probable que los repertorios conductuales que no han adquirido los niños (vestirse solos, prepararse algo sencillo de comer, brincar con un solo pie, etcétera) se deba a las conductas de sobreprotección que tienen con ellos sus cuidadores primarios, restringiendo la oportunidad de un buen manejo de su cuerpo y adquisición de conductas de autocuidado.

Porlo anterior, la evaluación es fundamental para detectar alteraciones o retrasos en su desarrollo e intervenir con la idea de que adquieran competencias que les permitan superar todos estos obstáculos.

A raíz de los resultados descritos, en el Centro Interdisciplinario de Educación Temprana Personalizada, UNAM-FES Iztacala (CIETEP, 2018), se ha propuesto mantener abierto un programa de atención para los niños con enfermedades crónicas con el objetivo de dar apoyo especializado para su óptimo desarrollo y proporcionar a los cuidadores principales un acompañamiento y favorecer la parentalidad positiva (Rodrigo, 2015).

\section{Referencias}

Acosta, J., Ramos, M., Zamora, L., \& Murillo, J. (2014). Prevalencia de infección de vías urinarias en pacientes hospitalizadas con amenaza de parto pre término. Rev. Ginecol Obstet Mex, 82, 737-743.

Ardila, R. (2013). Los orígenes del conductismo, Watson y el manifiesto conductista de 1913. Revista Latinoamericana de Psicología, 45(2), 315-319.

Arranz, P. Costa, M., Bayés, R., Cancio, H., Magallón, M., \& Fernández, F. (2000). El apoyo emocional en hemofilia. Madrid: Industrias Gráficas Sprint.

Bayley, N. (1977). Escalas Bayley de desarrollo infantil. España: TEA. Bayley, N. (1993). Bayley scales of infant development, 2a. ed.
USA: The Psychological Corporation.

Berger, K. S. (2001). The Developing Person through the Life Span. Nueva York: Worth Publishers.

Berger, K. S. (2007). Psicología del desarrollo. Infancia y adolescencia. Madrid: Editorial Médica Panamericana.

Blum Gordillo, B., Gordillo Paniagua, G., \& García Lozano, D. (2003). Psiconefrología. En G. Gordillo-Paniagua, R. A. Y. \& de la Cruz y J. Exeni (comp.), Nefrología pediátrica. Madrid: Elsevier Science.

Bronfenbrenner, U. (1987). La ecología del desarrollo humano. Experimentos en entornos naturales y diseñados. Barcelona: Paidós.

Chávez, R. (2003). Neurodesarrollo neonatal e infantil. Un enfoque multi-inter y transdisciplinario en la prevención del daño. México: Editorial Médica Panamericana.

CIETEP (2018). Centro Interdisciplinario de Educación Temprana Personalizada. http://medicina.iztacala.unam.mx/web cusi/cusi educacion temp.php

Craig, G. (2001). Desarrollo psicológico, 8a. ed. México: Pearson.

Eiser, C. (1996). Helping the Child with Chronic Disease. Themes and Directions. Clinical Child Psychology and Psychiatry, 1(4), 551-561.

Federación Mundial de Hemofilia (2012). Guías para el tratamiento de la Hemofilia. Canadá: Blackwell Publishing LTD. Recuperado el 27 de febrero de 2018 de http:// www1.wfh.org/publication/files/pdf-1513.pdf

Frankerburg, W., \& Dodds, J. (1990). Denver II Technical Manual. Denver: Denver Developmental Materials, Inc.

Garralda, M. E. (1994). Chronic Physical Illness and Emotional Disorder in Childhood. British Journal of Psychiatry, 164, 8-10

Gesell, A. (1985). Diagnóstico del desarrollo normal y anormal del niño. Evaluación y manejo del desarrollo neuropsicológico normal y anormal del niño pequeño y el preescolar. México: Paidós.

Gifre, M., \& Esteban, M. (2012). Consideraciones educativas de la perspectiva ecológica de Urie Bronfenbrenner. 15, 79-92.

Goldstein, G., Golan, G., Kenet, G., \& Martinowitz, U. (2000). From the Beginning. Group Therapy with Parents of Babies with Hemophilia. Haemophilia, 6(suppl 4), 417.

Gómez, A., Viguer, P., \& Cantero, M. (2003). Intervención temprana. Desarrollo óptimo de los 0 a 6 años. Madrid: Pirámide.

Graña, S. \& Rodríguez, O. M. (2000). Experience with Accompanying Parents. Haemophilia, 6(suppl 4), 418.

Houdé, O. (2006). La psicología del niño: Piaget revisado y superado. Madrid: Popular.

Huitrón, B., \& Alvarado, I. (1998). Valoración de conducta refleja y reacciones de defensa. Documento inédito del Centro Interdisciplinario de Educación Temprana Personalizada (cietep). México: FES-Iztacala, UNAM.

León, A. (1984). Desarrollo y atención del niño de 0 a 6 años. Primera parte: El desarrollo infantil humano. Aspectos generales. Universidad Estatal a Distancia.

Lozano, A., Betancourt, W., Turcios, L., Cueva, J. Ocampo, D., Portillo, C., \& Lozano, L. (2016). Sobrepeso y obesidad en el embarazo. Complicaciones y manejo. Archivos de Medicina, disponible en http://www.archivosdemedicina. com/medicina-de-familia/sobrepeso-y-obesidad-en-elembarazo-complicaciones-y-manejo.php?aid=11135 
Manual CTO de Medicina \& Cirugía (2016). Pediatría, 2a. ed. enarm-México: CTO Editorial.

McCarthy, D. (1991). Escalas McCarthy de aptitudes y psicomotricidad para niños. España: Publicaciones de Psicología Aplicada.

Midence, K. (1994). The Effects of Chronic Illness on Children and their Families. An Overview. Genetic, Social \& General Psychology Monographs, 120(3), 311-316.

Montealegre, R. (2016). Controversias Piaget-Vygotski en psicología del desarrollo. Acta Colombiana de Psicología, 19(1): 271-283.

Novak, J. D. (1988). Teoría y práctica de la educación. Alianza Universidad.

Osorio G., M. (2014). Escenarios de aplicación de la psicología de la salud relevancia del trabajo multidisciplinario. En M. Orozco y K. Caballero (eds.). Psicología latinoamericana. Experiencias, desafíos y compromisos sociales. México: ALFEPSI.

Osorio G., M. (2016). Repercusiones biopsicosociales en los padres que tienen un hijo con hemofilia. Revista de Investigación en Psicología,19(2): 111-121.

Osorio Guzmán, M., Olvera González, S., Bazán Riverón, G. E., \& Gaitán Fitch, R. C. (2016). Calidad de vida percibida por pacientes pediátricos con hemofilia y sus padres. Psicología y Salud. 26(1): 15-23. ISSN: 1405-1109.

Papalia, D. E., Wendkos, S., \& Duskin, R. (2009). Desarrollo humano, 11a. ed. México: Mc Graw-Hill.

Pedreira Massa, J. L. (1998). El niño enfermo crónico. Aspectos psicosomáticos y relacionales. Psiquis, 19(3), 89-96.

Perrin, J. M., \& Shonkoff, J. P. (2000). Developmental Disabilities and Chronic Illness. An Overview. En R. E. Behrman, R. M. Kliegman \& H. B. Jenson (eds.), Nelson Textbook of Pediatrics. Philadelphia: W. B. Saunders.

Reece, E. (2010). Obstetricia clínica, 3a. ed. Buenos Aires-México: Editorial Médica Panamericana.

Roberts, F. (1982). Perinatología. Cuidado del recién nacido y sus familia. Ediciones científicas. México: Prensa Médica Mexicana.

Rodrigo, M. (2015). Manual práctico de parentalidad positiva. Madrid: Síntesis.

Rogoff, B. (1993). Aprendices del pensamiento. El desarrollo cognitivo en el contexto social. Barcelona-México: Paidós.

Shaughnessy, J. J., Zechmeister, E. B., \& Zechmeister, J. S. (2007). Métodos de investigación en Psicología. México: McGraw-Hill

Tirado, T., Martínez, M., Covarrubias, P., López, M., Quesada, R., Olmos, A., \& Díaz-Barriga, F. (2010). Capítulo 3. Psicología del desarrollo. Psicología Educativa para afrontar los desafíos del siglo XXI. México: McGraw-Hill

Torralva, T., Cugnasco, I., Manso, M., Sauton, F., Ferrero, M., O'donnell, A., Duran, P., \& Carmuega, E. (1999). Desarrollo mental y motor en los primeros años de vida. Su relación con la estimulación ambiental y el nivel socio-económico. Arch.Argent.Pediatr, 97(5): 306 


\section{Meta-Análisis del Artículo}




\section{Dimensión Cuantitativa}

\section{Perfil de Evaluación entre pares}
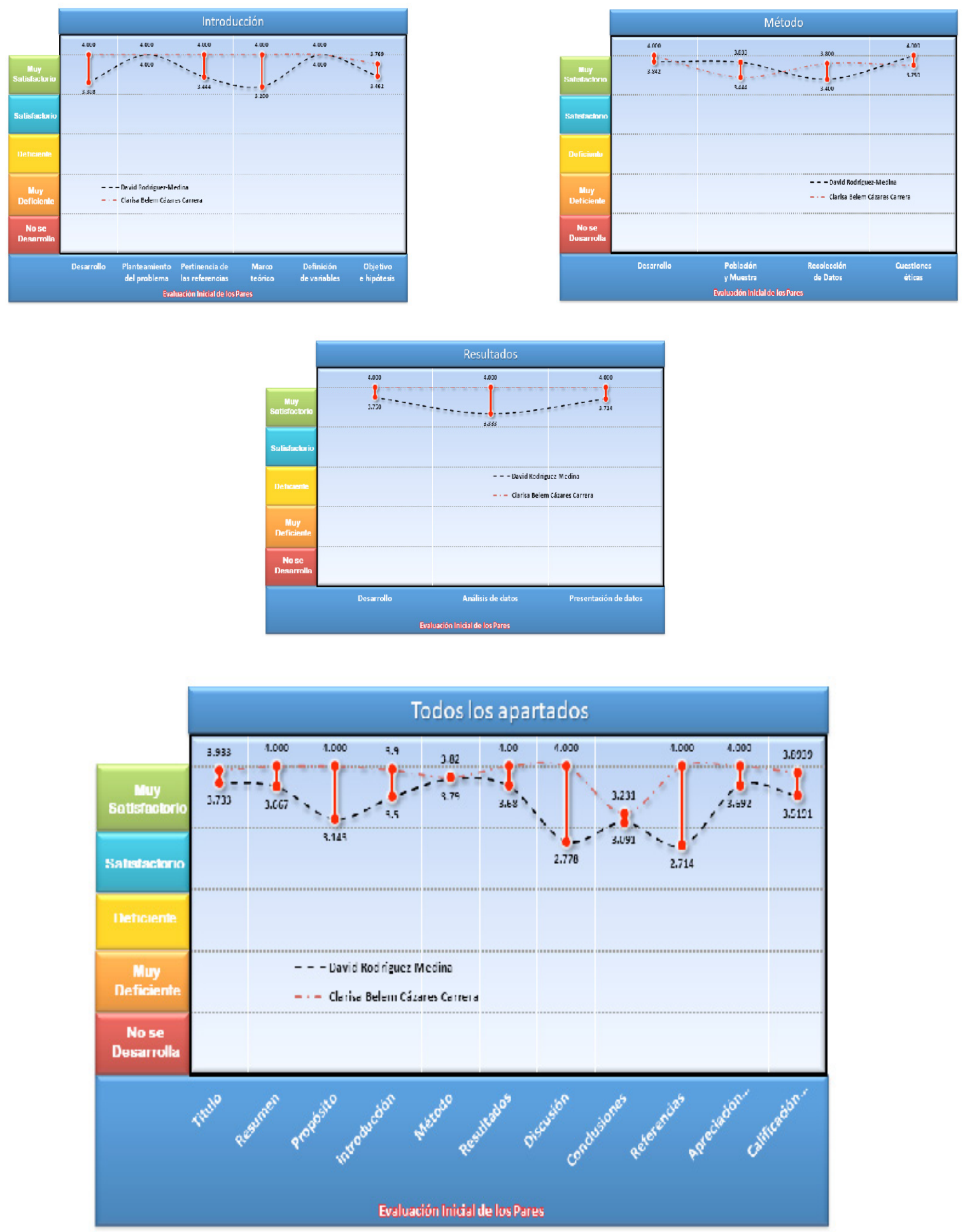


\section{Índice de Concordancia}

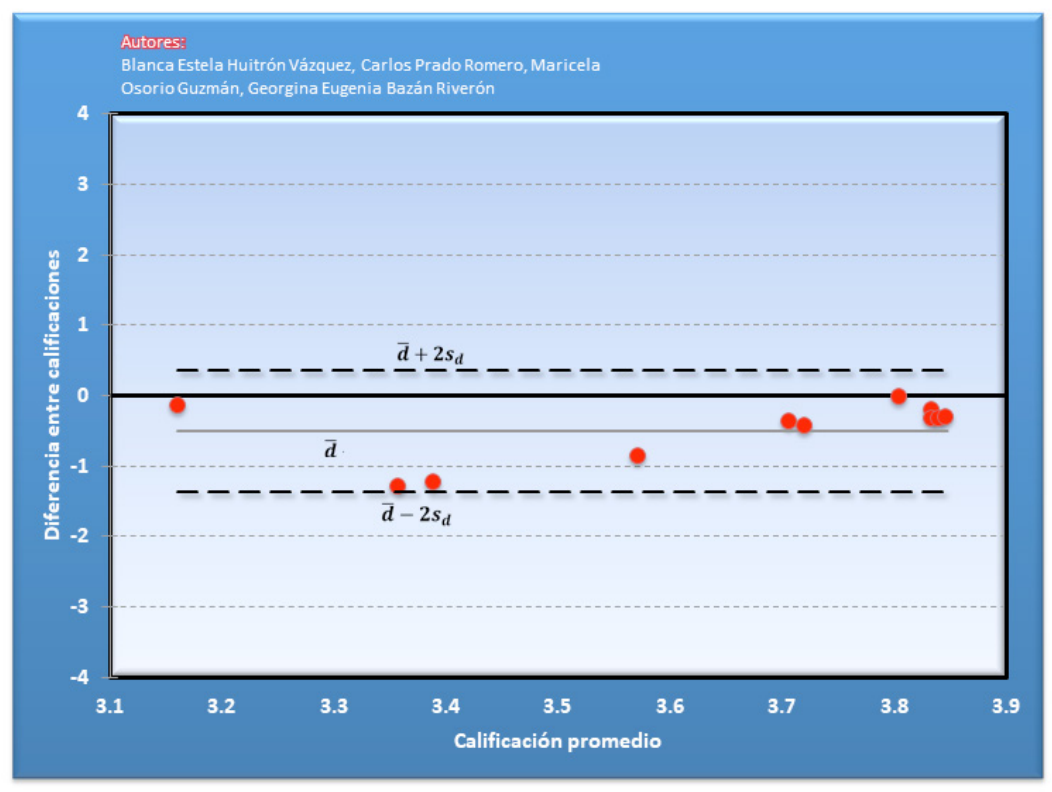

Índice de Acuerdo

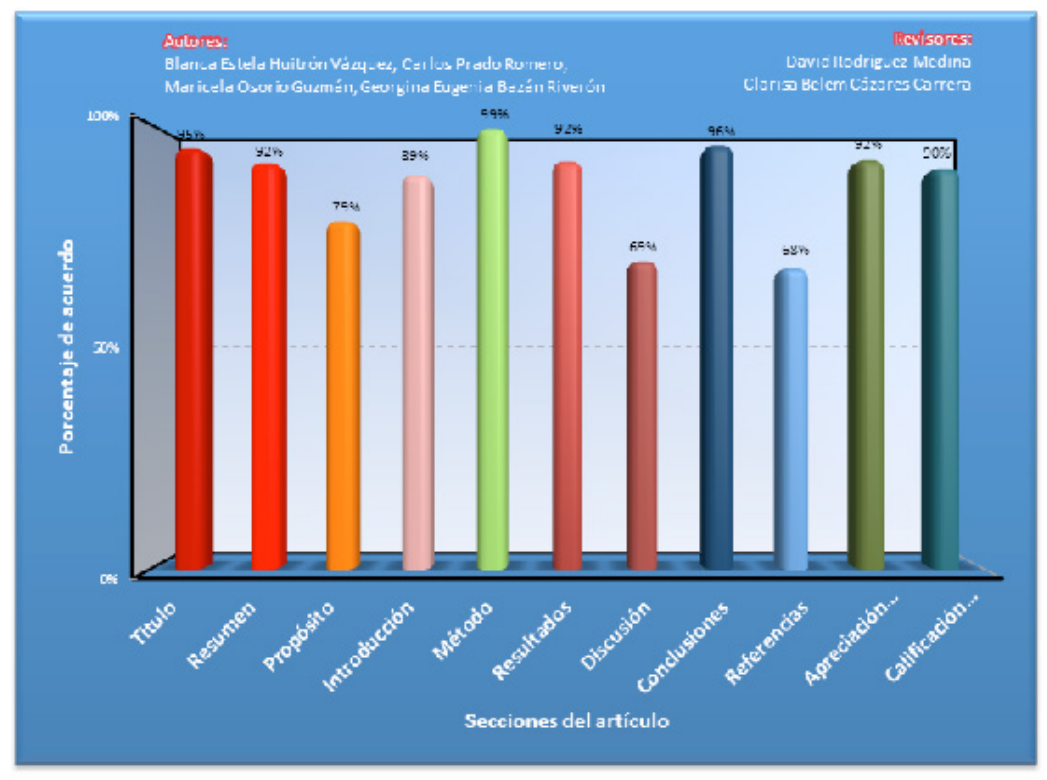




\begin{tabular}{|c|c|}
\hline Revisor 1 & Revisor 2 \\
\hline David Rodríguez-Medina & Clarisa Belem Cázares Carrera \\
\hline \multicolumn{2}{|c|}{ Título/Autoría } \\
\hline $\begin{array}{l}\text { El título sugiere que se considerarán costos-beneficios } \\
\text { clínicos. Podría modificarse de la siguiente manera: } \\
\text { Evaluación del desarrollo psicológico en pacientes pe- } \\
\text { diátricos con hemofilia. }\end{array}$ & $\begin{array}{l}\text { Lo único que agregaría al título es la palabra "desarro- } \\
\text { llo" para saber a que tipo de evaluación se refiere el es- } \\
\text { tudio. Faltan los correos de algunos autores. }\end{array}$ \\
\hline
\end{tabular}

Resumen

Se requieren ajustes puntuales en la descripción de la metodología

No tengo ningún comentario de mejora.

\section{Próposito del Estudio}

Se pueden agregar referencias recientes de las manifestaciones clínicas.

No tengo ningún comentario

\section{Introducción}

Se podrían agregar antecedentes de estudios similares.

No tengo ninguna sugerencia de mejora, me parece apropiada la manera en que se realizó.

\section{Método}

Explicar el procedimiento del muestreo.

No se describe la características de validez y confiabilidad de los instrumentos. Se entiende el tipo de muestra que es, pero no se describe tal cual.

\section{Resultados}

Se puede realizar una tabla que incluya la información cuantitativa con las descripciones de la evaluación que se redactan en la sección de resultados.

Los resultados son descriptivos y logran el objetivo del análisis 
Artículo Empírico | Importancia de la evaluación del neurodesarrollo... Huitrón-Vázquez, et al

\begin{tabular}{|c|c|}
\hline Revisor 1 & Revisor 2 \\
\hline \multicolumn{2}{|c|}{ Discusión } \\
\hline $\begin{array}{l}\text { Se sugieren indicar limitaciones y nuevas líneas de in- } \\
\text { vestigación. }\end{array}$ & Sin comentarios de mejora. \\
\hline \multicolumn{2}{|c|}{ Conclusiones } \\
\hline $\begin{array}{l}\text { Se debe cuidar la utilidad del estudio colocándolo en su } \\
\text { justa dimensión. }\end{array}$ & $\begin{array}{l}\text { No se proponen investigaciones futuras, sólo aplicacio- } \\
\text { nes. }\end{array}$ \\
\hline \multicolumn{2}{|c|}{ Referencias } \\
\hline Revisar cómo citar las publicaciones de revistas. & Sin comentarios de mejora. \\
\hline
\end{tabular}

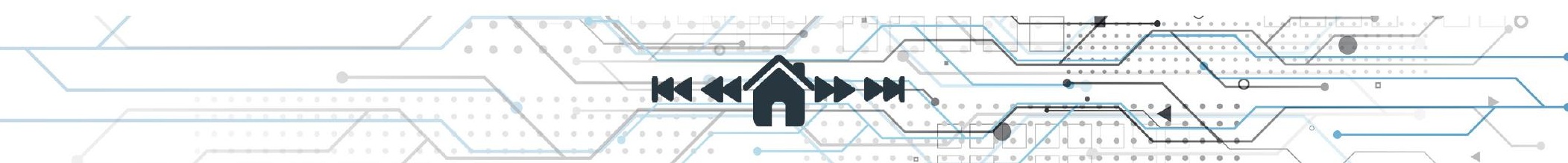




\section{EDITORIAL}

